\title{
Predicting the Dynamic Behavior of Materials with a Nonlinear Modified Voigt Model
}

\author{
Marc Delphin Monsia (Corresponding author) \\ Département de Physique, Université d'Abomey-Calavi \\ Abomey-Calavi, 09 B.P. 305, Cotonou, Bénin
}

Tel: 229-95-568-187Ｅ-mail: monsiadelphin@yahoo.fr

Yélomè Judicaël Fernando Kpomahou

Doctorant au Département de Physique

Université d'Abomey-Calavi, Abomey-Calavi, Bénin

Tel: 229-95-799-377 E-mail: kpomaf@yahoo.fr

Received: January 29, 2012

Accepted: February 10, 2012 Published: Apri1 1, 2012

doi:10.5539/jmsr.v1n2p166

URL: http://dx.doi.org/10.5539/jmsr.v1n2p166

\begin{abstract}
In this paper, a one-dimensional nonlinear modified and extended Voigt model with constant material parameters is formulated to represent mathematically the time deformation behavior of a variety of viscoelastic materials. A binomial law is used as a nonlinear elastic force function. Numerical illustrations performed show that the hyperlogistic-type solution obtained is very useful to reproduce any S-shaped experimental curve.
\end{abstract}

Keywords: Binomial elastic force function, Hyperlogistic-type function, Lambert-type equation, Riccati equation, Viscoelasticity, Voigt model

\section{Introduction}

The understanding of rheological properties of materials is of a great interest in many fields of science and engineering. These properties are necessary to be known and predicted before design and optimization process. Theoretical models that describe material responses subjected to deformations or forces appear then as important analytical tools in mechanics. In the characterization of materials, these are often considered as purely elastic or viscoelastic materials following that the time effect is neglected or taken into account. But, it is well known that real materials are time and history-dependent, to say, viscoelastic materials. Viscoelastic materials exhibit both combined elastic and viscous material behaviors in which the constitutive stress-strain equation is time-dependent. In the modeling of rheological materials, the simplest theory consists of a linear viscoelastic model. However, the well known established linear viscoelastic theory is only applicable for small deformations or low stresses. Since mechanical properties of materials are in general nonlinear in cases of intermediate and high deformations and stresses, nonlinear viscoelastic theories are required. Strictly speaking, viscoelastic materials are characterized by elastic, viscous and inertial contributions. These contributions are highly nonlinear and involve more complex material responses under mechanical solicitations. Therefore, an adequate constitutive equation must be nonlinear and must relate mathematically stress, strain and their higher time derivatives (Bauer, et al., 1979; Bauer, 1984). Since also linear viscoelastic theory is usually described in the Boltzmann single integral representation or in the differential form, one can extend these equations to higher order stress or strain terms in order to model material nonlinearities. From a mathematics point of view, the integral representation of viscoelastic constitutive equation is more difficult to perform than the differential form. In that perspective, several studies of different complexities have been performed to describe viscoelastic material functions. Many successful predictive models are also shown to be based on extension of classical linear rheological models to finite deformations (Monsia, 2011a, 2011b, 2011c, 2011d, 2011e). Monsia (2011a), recently, using a second-order elastic spring in series with a classical Voigt element, which is an extended form of the standard linear solid to finite strains, constructed a hyperlogistic-type equation to reproduce the nonlinear time-dependent stress response of some viscoelastic materials. Monsia (2011b) again, developed a single 
differential constitutive equation derived from a nonlinear standard solid model consisting of a polynomial elastic spring in series with a classical Voigt element for the prediction of time-dependent nonlinear stress of a class of viscoelastic materials. In (Monsia, 2011c) the author formulated a nonlinear four-parameter rheological Voigt model consisting of a nonlinear Voigt element in series with a classical linear Voigt element with constant material coefficients for representing the nonlinear stiffening response of the initial low-load portion and the softening, that is to say, the S-shaped mechanical behavior of some viscoelastic materials. Recently, Monsia (2011e) developed a simple nonlinear generalized Maxwell fluid model consisting of a nonlinear spring connected in series with a nonlinear dashpot obeying a power-law with constant material coefficients for representing accurately the time-dependent behavior of some viscoelastic materials. However, in many viscoelastic models, such as the previous models, except the model (Monsia, 2011d), the inertia of the system under study was neglected. Moreover, there are only a few theoretical models that consider both nonlinear elastic properties and nonlinear viscous properties of materials simultaneously in their formulation, due to mathematical complexities. To overcome these above mentioned difficulties, Bauer (1984) studying the rheological properties of arterial walls, developed an approach based on the classical Voigt model. This theory (Bauer, 1984) consists to decompose the total stress acting on the material as the sum of three components, that is, the elastic, viscous and inertial stresses. This method has been after used by many authors (Armentano, et al., 1995; Gamero, et al., 2001; Monsia, et al., 2009) for a complete characterization of arterial behavior. In (Monsia, et al., 2009), following the Bauer's approach (1984), elastic, viscous and inertial stresses are expanded in power series of strain. Monsia (2011d) using also the Bauer's method (1984) and expressing the elastic stress as an asymptotic expansions in powers of deformation, the viscous stress as a first time derivative of a similar asymptotic expansions in powers of deformation, and the inertial stress as a second time derivative of a similar asymptotic expansions in powers of deformation, developed a hyperlogistic equation that represents successfully the time-dependent mechanical properties of a variety of viscoelastic materials. Monsia (2011f) using again the Bauer's approach (1984), proposed a nonlinear rheological model based on a substitution of linear elastic and damping forces in Voigt model by nonlinear elastic force and damping force with inclusion of a body for providing a theoretical basis to empirical exponential or logistic formulas used by several authors for fitting the stress-strain experimental data of arteries. Recently, Monsia $(2011 \mathrm{~g}$ ) by considering the same approach (Bauer 1984), developed a hyper-exponential type model to describe the deformation behavior of a variety of materials. The model (Monsia, 2011g) was an important modification and extension to finite deformation of the classical linear Voigt model. In (Monsia, 2011g), the nonlinear elastic force function has been expressed as a simple hyperbolic law, and the viscous and inertial stresses as a first and a second time derivatives of a similar law, respectively. The obtained evolution equation of the deformation is a Lambert-type equation that has been solved in closed-form solution, by using suitable boundary conditions, in a hyper-exponential type function of time. More recently, Monsia (2012) using also the same hyperbolic law as elastic force function and applying the Bauer's theory (1984), developed successfully a hyperlogistic-type model that appeared powerful to describe the time dynamic response of viscoelastic materials under a constant applied load, to say, the nonlinear deformation behavior of viscoelastic materials during creep tests. In this paper, by applying once more the Bauer's theory (1984), a one-dimensional nonlinear modified and extended Voigt model with constant material parameters is formulated to represent mathematically the time deformation behavior of a variety of viscoelastic materials. A binomial law is used as a nonlinear elastic force function. The resulting evolution equation of the time dependent deformation is a Lambert-type nonlinear ordinary differential equation that is solved in analytic solution by considering suitable boundary conditions. Numerical illustrations performed show that the hyperlogistic-type solution obtained is very useful to reproduce any S-shaped experimental data.

\section{Formulation of the Mechanical Model}

\subsection{Theoretical Considerations}

In this part the one-dimensional theoretical model proposed will be described and the governing equations including elastic, viscous and inertial nonlinearities will be also derived. To that end, we consider the Bauer's theory (1984) as formulated previously by Monsia (2011g; 2012). Thus, by superposing the elastic, viscous and inertial stresses, for a nonlinear elastic force function $\varphi(\varepsilon)$, where the deformation $\varepsilon(t)$ is a scalar function of time $t$, it arises the following differential evolution equation (Monsia ,2011g; Monsia, 2012)

$$
\ddot{\varepsilon} \frac{d \varphi}{d \varepsilon}+\dot{\varepsilon}^{2} \frac{d^{2} \varphi}{d \varepsilon^{2}}+\frac{b}{c} \dot{\varepsilon} \frac{d \varphi}{d \varepsilon}+\frac{a}{c} \varphi(\varepsilon)=\frac{1}{c} \sigma_{t}
$$


where the dot over a symbol denotes a differentiation with respect to time $t$ and the inertial coefficient $c$ is different from zero. The coefficients $a$ and $b$ are respectively the stiffness and viscosity modules. These all three coefficients are time independent material parameters. The scalar function $\sigma_{t}$ denotes the exciting stress acting on the material studied. At this stage of modeling, it is required to specify the nonlinear elastic force function $\varphi(\varepsilon)$. A basic principle that governs the Bauer's theory is that, for small deformations, the function $\varphi(\varepsilon)$ should reproduce the well known linear elastic force function. In this regard, the function $\varphi(\varepsilon)$ is empirically formulated, in the present work, as the following binomial function

$$
\varphi(\varepsilon)=(1-\varepsilon)^{m}
$$

where the real number $m$ is a material parameter. Therefore, using Equation (2), Equation (1) gives

$$
\sigma_{t}=a(1-\varepsilon)^{m}-m b \dot{\varepsilon}(1-\varepsilon)^{m-1}+m(m-1) c \dot{\varepsilon}^{2}(1-\varepsilon)^{m-2}-m c \ddot{\varepsilon}(1-\varepsilon)^{m-1}
$$

Equation (3) represents analytically in the single differential form the relation between the exciting stress $\sigma_{t}$ and the resulting strain $\varepsilon$. Equation (3) denotes a second-order nonlinear ordinary differential equation in $\varepsilon$ for a given exciting stress $\sigma_{t}$. Note that in the case where the parameter $m$ is negative, the function $\varphi(\varepsilon)$ tends towards infinity, to say, stiffens for $\varepsilon \rightarrow 1$.

\subsection{Dimensionalization}

The strain $\varepsilon(t)$ is a dimensionless quantity. Then in Equation (3) the coefficients have the following dimensions. Let now $M, L$ and $T$ denote the mass, length and time dimension respectively, the dimension of the stress varies as $M L^{-1} T^{2}$. Therefore, the dimension of $a$ varies as $M L^{-1} T^{-2}$, that of $b$ varies as $M L^{-1} T^{-1}$, and that of $c$ varies as $M L^{-1}$ (mass per unit length).

\subsection{Solution using a stress $\sigma_{t}=0$}

\subsubsection{Evolution Equation of the Deformation $\varepsilon(t)$}

In the absence of exciting stress $\left(\sigma_{t}=0\right)$, the internal dynamics of the viscoelastic material under study is expressed by the following governing evolution Lambert-type equation

$$
a(1-\varepsilon)^{m}-m b \dot{\varepsilon}(1-\varepsilon)^{m-1}+m(m-1) c \dot{\varepsilon}^{2}(1-\varepsilon)^{m-2}-m c \ddot{\varepsilon}(1-\varepsilon)^{m-1}=0
$$

This can be also rewritten as

$$
\ddot{\varepsilon}-(m-1) \dot{\varepsilon}^{2}(1-\varepsilon)^{-1}+\frac{b}{c} \dot{\varepsilon}-\frac{a}{m c}(1-\varepsilon)=0
$$

or

$$
\ddot{\varepsilon}-(m-1) \frac{\dot{\varepsilon}^{2}}{1-\varepsilon}+\frac{b}{c} \dot{\varepsilon}-\frac{a}{m c}(1-\varepsilon)=0
$$

or finally

$$
\ddot{\varepsilon}-(m-1) \frac{\dot{\varepsilon}^{2}}{1-\varepsilon}+\lambda \dot{\varepsilon}-\frac{\omega_{o}^{2}}{m}(1-\varepsilon)=0
$$

where $\lambda=\frac{b}{c}, \omega_{o}^{2}=\frac{a}{c}$, and $c$ different from zero. Equation (5) represents the Lambert-type evolution equation of the material under study.

2.3.2 Solving Time-Deformation Equation

With the help of the following change of variable 


$$
x=1-\varepsilon
$$

Equation (5) transforms in the form

$$
\ddot{x}+(m-1) \frac{\dot{x}^{2}}{x}+\lambda \dot{x}+\frac{\omega_{o}^{2}}{m} x=0
$$

Substituting

$$
f=\frac{\dot{x}}{x}
$$

into Equation (7) now leads to the expression

$$
\dot{f}=-m f^{2}-\lambda f-\frac{\omega_{o}^{2}}{m}
$$

Equation (9) is a first-order nonlinear Riccati differential equation for the variable $f$ that possesses the strain rate dimension. Due to the fact that $f$ is defined as a frequency, the nonlinearity parameter $m$ must be negative. Using suitable boundary conditions that satisfy the dynamics of the viscoelastic material under study, that is to say

$$
t \rightarrow 0, \lim f(t)=f_{o},
$$

and

$$
t \rightarrow+\infty, \lim f(t)=0
$$

the following explicit analytical solution, by integration, is obtained

$$
x(t)=x_{\max }[1+q \exp (-\lambda \delta t)]^{-\frac{1}{n}}
$$

in which, $\delta=\sqrt{1-4 \frac{\omega_{o}^{2}}{\lambda^{2}}}, f_{2}=-\frac{\lambda}{2 m}(1-\delta), q=\frac{f_{o}}{f_{2}-f_{o}}, n=\frac{\lambda \delta}{f_{2}}$, and the condition $t \rightarrow+\infty$, $\lim x(t)=x_{\max }=K$

By considering Equation (6) one can easily obtain the desired strain

$$
\varepsilon(t)=1-K[1+q \exp (-\lambda \delta t)]^{-\frac{1}{n}}
$$

Equation (11) gives the strain versus time variation in the viscoelastic material under consideration. It represents mathematically the time dependent deformation behavior of the material studied as a hyperlogistic-type model that is known to be powerful to reproduce any S-shaped curve.

\section{Numerical Results and Discussion}

In this section some numerical illustrations are presented to show the predictive ability of the model to reproduce the mechanical response of the material considered. The dependence of the strain versus time curve on material parameters is also discussed. Note that the numerical illustrations are investigated at the fixed value $K=-1$. In this work, the function $\varphi(\varepsilon)$ is given by a binomial expression. This spring force function is very well suited by the fact that for $0<\varepsilon<1, \varphi(\varepsilon)$ can be expanded in power series of deformation, and agrees then very well with the polynomial function of deformation used by Bauer (1984). Another features that involve the choice of this 
binomial law, is that it allows, contrary to previous restoring force function (Monsia, 2011g; 2012), to assure or to control the nonlinearity of the model via an explicit material parameter, to say, via the coefficient $m$. If the parameter $m=1$, Equation (5) or (7) becomes a simple linear ordinary differential equation. For $\varepsilon>>1$, the factor $1-\varepsilon$ reduces to the term $-\varepsilon$, so that Equation (5) becomes identical to the fundamental Lambert-type evolution equation of the deformation obtained in (Monsia, 2011d). Therefore, the previous model (Monsia 2011d) can be interpreted as a special case of the present model. Note that it is possible to control the magnitude of strain $\varepsilon$ with the help of a finite extensibility coefficient $s$ so that, the function $\varphi(\varepsilon)=(1-s \varepsilon)^{m}$. But, this study will be done as a subsequent work. For several applications using viscoelastic materials linear models are inapplicable. Then, the formulation of the time dependent consistent nonlinear deformation model at large strains becomes a prerequisite in many branches of science and engineering applications. By applying the Bauer's theory (1984) that is well-suited to account for material nonlinearities, a one-dimensional nonlinear four-parameter rheological model is developed. By expanding the elastic force function in term of a generalized binomial expression, the viscous damping and the inertial forces in terms of the first and second time derivatives of a similar generalized binomial law, we have successfully described the nonlinear time dependent behavior of the mechanical system studied as a hyperlogistic-type function, that appeared useful to reproduce any sigmoid curve. With four material parameters, the present model becomes more flexible for data fitting than (Monsia $2011 \mathrm{~g}$ ) that contains three material parameters.

\section{Conclusions}

The one-dimensional classical Voigt model is modified and extended to finite deformation in order to take into consideration elastic, viscous and inertial nonlinearities simultaneously. The use of a binomial elastic spring force function allows controlling the nonlinearity of the model by means of an explicit material parameter. The model is applicable for describing the time deformation behavior of some viscoelastic materials. The material response is represented as a hyperlogistic-type function that is useful to reproduce any S-shaped curve. Numerical examples showed the predictive capability of the model and its sensitivity to material parameters.

\section{References}

Armentano, R. L., Barra, J. G., Levenson, J., Simon, A., \& Pichel, R. H. (1995). Arterial wall mechanics in conscious dogs. Assessment of viscous, inertial, and elastic moduli to characterize aortic wall behaviour. Circ Res., 76, 468-478.

Bauer, R. D., Busse, R., Schabert, A., Summa, Y., \& Wetterer, E. (1979). Separate determination of the pulsatile elastic and viscous forces developed in the arterial wall in vivo. Pflügers. Arch., 380, 221-226. http://dx.doi.org/10.1007/BF00582900

Bauer, R. D. (1984). Rheological approaches of arteries. Biorheology. Suppl., I, 159-167.

Gamero, L. G., Armentano, R. L., Barra, J. G., Simon, A., \& Levenson, J. (2001). Identification of Arterial Wall Dynamics in Conscious Dogs. Exp. Physiol., 86, 519-528. http://dx.doi.org/10.1113/eph8602172

Monsia, M. D. (2011a). A Hyperlogistic-type Model for the Prediction of Time-dependent Nonlinear Behavior of Viscoelastic Materials. Int. J. Mech. Eng., 4, 1-4.

Monsia, M. D. (2011b). A Nonlinear Generalized Standard Solid Model for Viscoelastic Materials. Int. J. Mech. Eng., 4, 11-15.

Monsia, M. D. (2011c). A Modified Voigt Model for Nonlinear Viscoelastic Materials. Int. J. Mech. Eng., 4, 17-20.

Monsia, M. D. (2011d). Lambert and Hyperlogistic Equations Models for Viscoelastic Materials: Time-dependent Analysis. Int. J. Mech. Eng., 4, 5-10.

Monsia, M. D. (2011e). A Simplified Nonlinear Generalized Maxwell Model for Predicting the Time Dependent Behavior of Viscoelastic Materials. World Journal of Mechanics, 1, 158-167. http://dx.doi.org/10.4236/wjm.2011.13021

Monsia, M. D., Massou, S., \& Moussiliou, S. (2009). Nonlinear Mathematical Model for Viscoelastic Behavior of Arterial Wall. Rev. CAMES-Série A, 08, 12-17.

Monsia, M. D. (2011f). A Riccati Pressure-Length Equation for Arterial Walls Using a Nonlinear Voigt Model. International Journal of Computational Intelligence and Bioinformatics, 3, 71-82.

Monsia, M. D. (2011g). Modeling the Nonlinear Rheological Behavior of Materials with a Hyper-Exponential Type Function. Mechanical Engineering Research, 1(1), 103-109. http://dx.doi.org/10.5539/mer.v1n1p103 
Monsia, M. D. (2012). A Nonlinear Mechanical Model for Predicting the Dynamics Response of Materials under a Constant Loading. Journal of Materials Science Research, 1(1), 90-100. http://dx.doi.org/10.5539/jmsr.v1n1p90

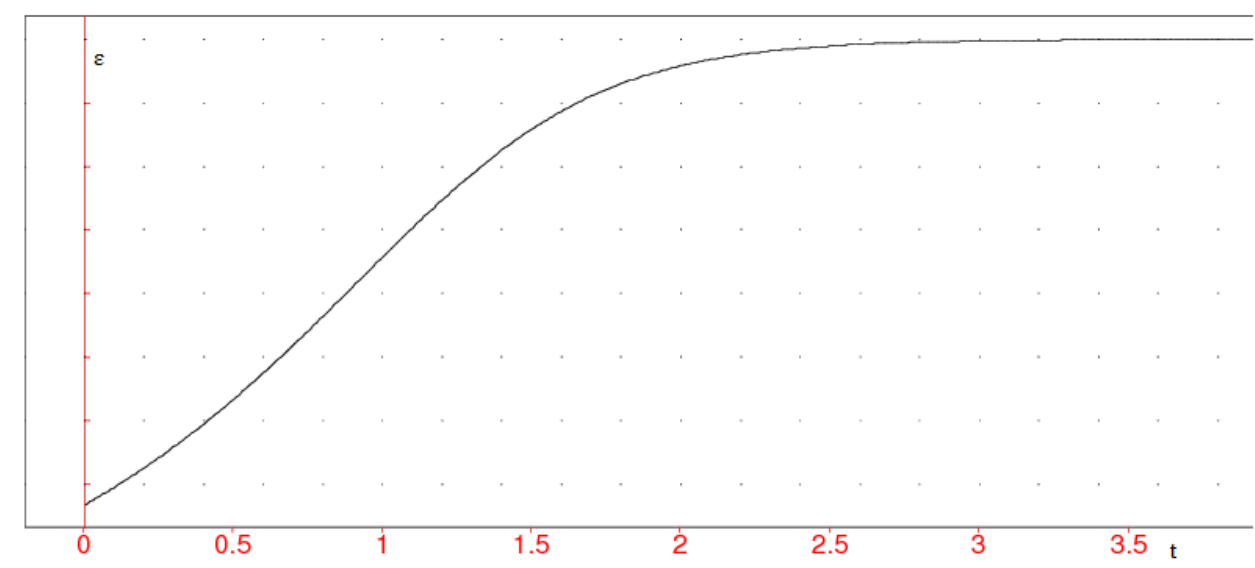

Figure 1. Typical strain versus time curve showing an asymptotical value

Figure 1 illustrates the typical time dependent strain behavior with an increase until an asymptotical value, resulting from Equation (11) with the fixed value of coefficients at $m=-1, \lambda=4.9275, \omega_{o}=2, f_{o}=1$. It can be seen from Figure 1 that the model is capable to reproduce mathematically and accurately the typical exponential deformation of some viscoelastic materials (Monsia, 2011g). The strain versus time curve is nonlinear, with a nonlinear beginning initial region. The plotting illustrates then the S-shaped deformation behavior of the viscoelastic material under study.

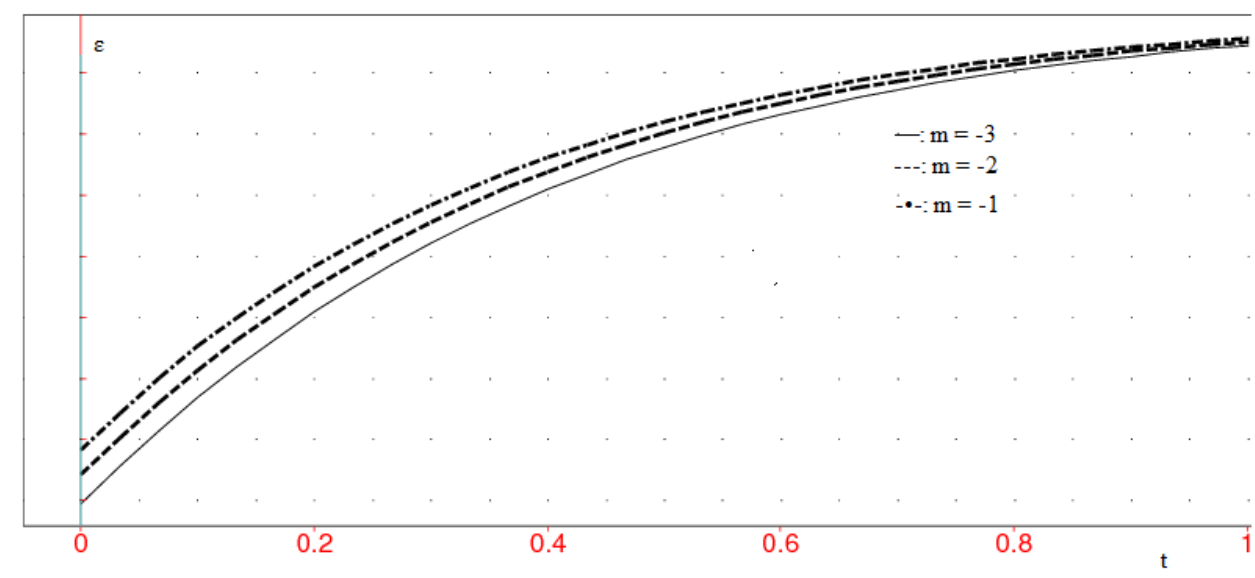

Figure 2. Strain time curve with three different values of $m$

Figure 2, 3, 4 and 5, demonstrate the effects of material coefficients on the strain. The effects of these coefficients are studied by varying one coefficient while keeping the other three constant. In Figure 2 is shown the dependence of the strain versus time relationship on the nonlinearity parameter $m$. The graph indicates that the initial value of the strain increases with increasing $m$ so that, the strain value increases in the early period of time before to reach the peak asymptotical value. However, an increasing $m$, has no significant effect on the time needed to reach the maximum strain value. The solid line corresponds to $m=-3$, the dashed line to $m=-2$, and the dash-dot line to $m=-1$. The other parameters are $\lambda=4.9275, \omega_{o}=2, f_{o}=0.1$. 


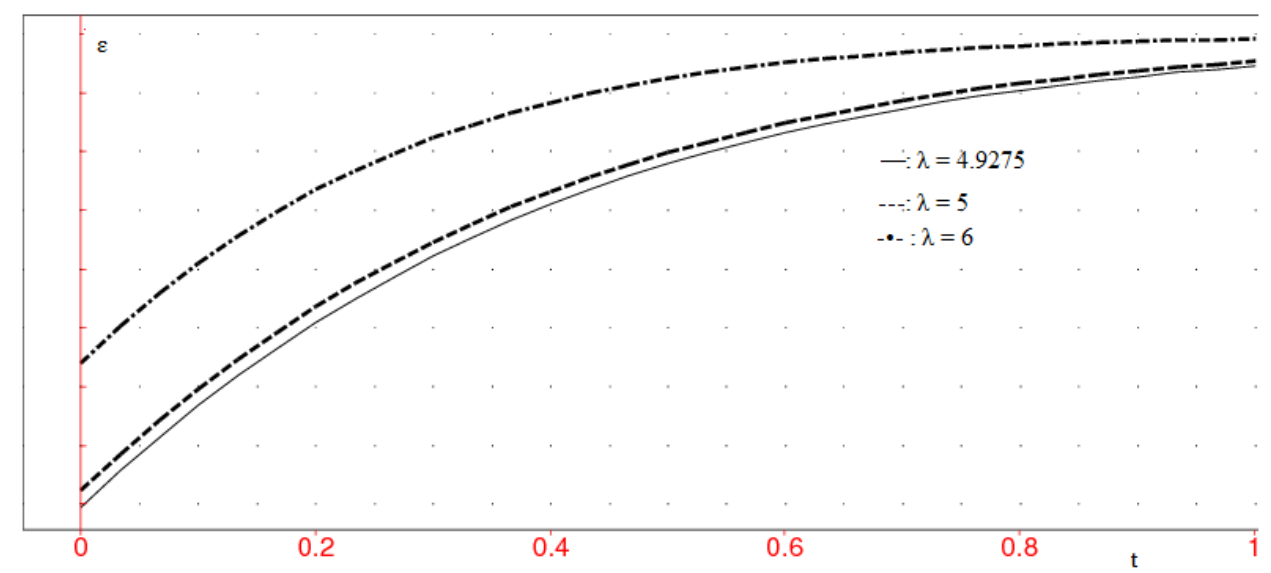

Figure 3. Strain time curves showing the effect of the coefficient $\lambda$

The strain curves at various values of the viscous damping term $\lambda$ for the material under consideration are shown in Figure 3. An increasing $\lambda$, increases the initial value of the strain so that, the strain value increases fast and significantly in the early period of time before to attain the peak asymptotical value. An increasing $\lambda$, reduces also the time required to attain the maximum asymptotical strain. The solid line corresponds to $\lambda=4.9275$, the dashed line to $\lambda=5$, and the dash-dot line to $\lambda=6$. The other parameters are $m=-3$, $\omega_{o}=2, f_{o}=0.1$.

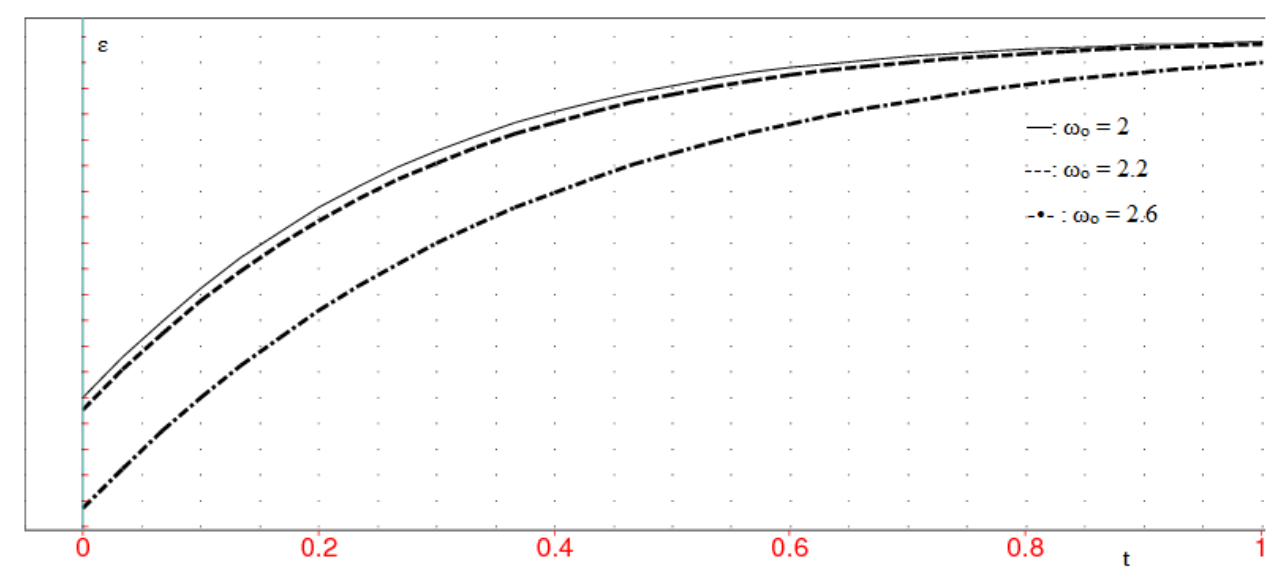

Figure 4. Strain versus time curves showing the effects of the natural frequency $\omega_{o}$

Figure 4 exhibits the dependence of the strain versus time curve on the natural frequency $\omega_{o}$. The graph indicates that an increase $\omega_{o}$, decreases the initial value of the strain so that, the strain value decreases fast and significantly in the early period of time before to attain the peak asymptotical value. An increase $\omega_{o}$, increases also the time needed to reach the maximum asymptotical strain. It is worth noting that the effects of the viscous coefficient $\lambda$ and the frequency coefficient $\omega_{o}$ affect the strain versus time curve in opposite directions. The solid line corresponds to $\omega_{o}=2$, the dashed line to $\omega_{o}=2.2$, and the dash-dot line to $\omega_{o}=2.6$. The other parameters are $m=-3, \lambda=6, f_{o}=0.1$. 


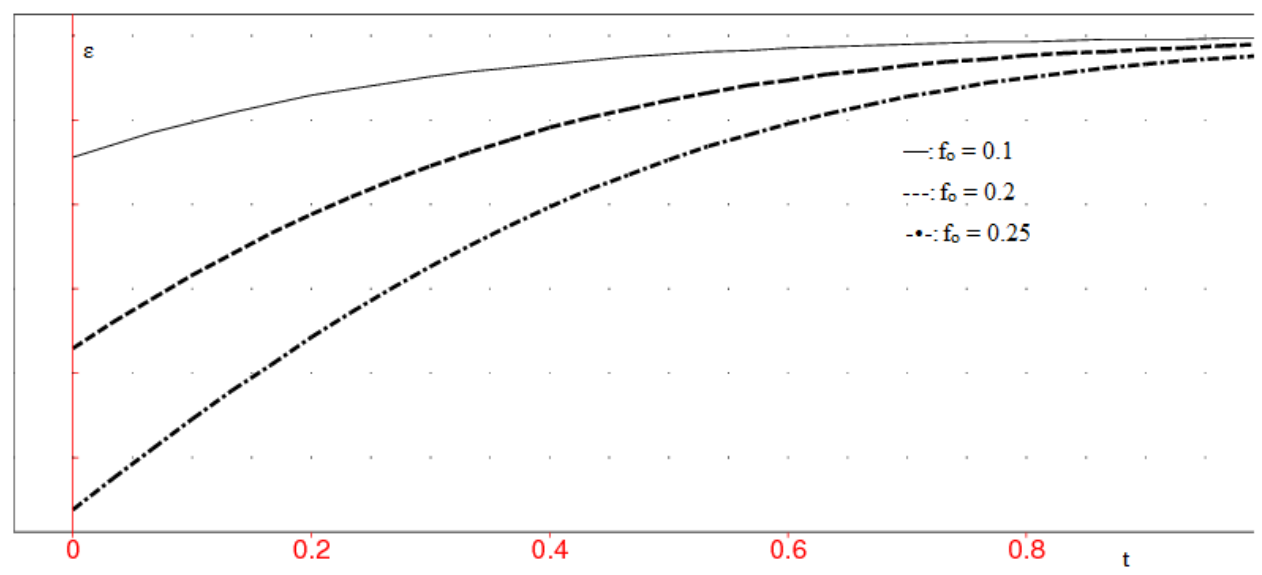

Figure 5. Strain time curves for various values of the coefficient $f_{o}$

Figure 5 illustrates how the coefficient $f_{o}$ affects the strain versus time curve. An increasing $f_{o}$, decreases the initial value of the strain and increases the time necessary to reach the peak asymptotical strain. The curves become also more linear with decreasing $f_{o}$. The solid line corresponds to $f_{o}=0.1$, the dashed line to $f_{o}=0.2$, and the dash-dot line to $f_{o}=0.25$. The other parameters are $m=-3, \lambda=6, \omega_{o}=2.2$. 